\title{
Comparison of efficacy and safety of intravenous iron sucrose and intramuscular iron sorbitol therapy in patients with anemia during pregnancy in a developing country
}

\author{
Harsha Shailesh Gaikwad', Anu Sindhu², Nivedita Sarda ${ }^{3}$ \\ ${ }^{1}$ Professor, ${ }^{2}$ Ex Resident, ${ }^{3}$ Ex-Senior Specialist, Department of Obstetrics and Gynaecology, Vardhman Mahavir \\ Medical College and Safdarjang Hospital, New Delhi, India
}

A B S T R A C T

Background: Iron deficiency anemia due to poor nutrition is estimated to affect nearly two thirds of all pregnant women in developing countries particularly in India. The responsible constellation of factors producing iron deficiency anemia generally precede the pregnancy, which include deficient diet in iron content coupled with menstrual losses, generally a rapid succession of pregnancies and inadequate supplemental iron are a few to be blamed for a high incidence of anemia in such population. Aims and Objectives: To compare the efficacy and safety of iron sucrose versus iron sorbitol therapy in pregnant anemic patients. Materials and Methods: Two-hundred antenatal women attending the out-patient department having hemoglobin levels of $8 \mathrm{gm} \%$ or less were recruited in the study and were randomized into two groups for receiving intravenous and intramuscular iron therapy. Results and Conclusions: There was a significant improvement in the haematological parameters and no major side effects developed in the intravenous iron sucrose group. Though there was a significant improvement in the haematological parameters after intramuscular iron sorbitol therapy also but none of the cases reached the target level of haemoglobin during the observation period.

Key words: Anemia, Iron sucrose, Pregnancy

\section{INTRODUCTION}

Iron deficiency anemia due to poor nutrition is estimated to affect nearly two thirds of the pregnant women in developing countries particularly in India. ${ }^{1}$ The responsible constellation of factors producing iron deficiency anemia generally precedes the pregnancy, including diet poor in iron content coupled with menstrual losses, a rapid succession of pregnancies and inadequate supplemental iron are a few to be blamed for a high incidence of anemia in such population. Poor knowledge of dietary requirements during pregnancy and low socioeconomic status also contribute to developing anemia. Most such women begin their pregnancy with partially or completely depleted iron reserves, which further gets worsened as during pregnancy the demand for iron is greatly increased.

Most of the contemporary studies on the topic reveal that despite the Iron Folic Acid (IFA) program, which has been running for more than 30 years, anemia in pregnancy remains a major problem in India.

Over the past many years, oral, intramuscular and intravenous preparations of iron have been used for treating anemia during pregnancy. Even though each therapy has its own implications and side effects during pregnancy, the first choice in the treatment of iron deficiency anemia for almost all patients is oral iron replacement because of its easy availability, effectiveness, safety, and lower cost. 
Parenteral iron therapy is reserved for patients in whom oral treatment fails.

\section{Study design}

It was a prospective randomized study.

\section{MATERIAL AND METHODS}

A total of 200 pregnant women attending the out patient department between gestational age 24 to 32 weeks having hemoglobin levels of $8 \mathrm{gm} \%$ or less were enrolled in the study out of 25,000 pregnant cases. Institutional ethical clearance was obtained before initiation of study. The recruitment was stopped after 200 cases were enrolled as was required by the institutional review board and the ethics committee, since this was a part of post graduate dissertation project which needed to be completed during a stipulated time frame of not more than 18 months.

\section{Duration of study}

12 months, from July 2011 to June 2012

\section{Inclusion criteria}

Pregnant women of gestational age within 24 weeks and 32 weeks with iron deficiency anemia diagnosed on peripheral smear and who consented to the study.

\section{Exclusion criteria}

Women with liver disease, renal disease, dimorphic anemia, hemolytic anemia, any infective conditions, multi-fetal gestation, severe anemia $(\mathrm{Hb} \leq 5 \mathrm{gm} \%)$ and those who had earlier received blood transfusion or parenteral iron treatment were excluded from study.

These women were randomised into two groups as Group A and Group B, to be designated as intravenous group and intramuscular group respectively. Each group had 100 women. The following tests were done on each woman, peripheral smear for type of anemia, evidence of hemolysis, reticulocyte count, hematocrit, mean corpuscular hemoglobin concentration (MCHC), mean corpuscular volume (MCV), mean corpuscular hemoglobin $(\mathrm{MCH})$ and serum ferritin level estimation.

The total iron requirement for each recruit was calculated by the following formula

Total iron requirement $=$ Iron deficit + amount of iron required to replenish stores

Iron deficit $(\mathrm{mg})=$ Body weight $(\mathrm{kg}) \times$ Hb deficit $\times 0.3$

Hemoglobin $(\mathrm{Hb})$ deficit $=\quad$ Target $\mathrm{Hb}-$ Initially measured $\mathrm{Hb}$

\section{Target $\mathrm{Hb}$ concentration $=$ \\ $11 \mathrm{gm} \%$}

Iron required to replenish stores $(\mathrm{mg})=$ Body weight $(\mathrm{kg}) \times 10$

Intravenous iron sucrose infusion was prepared by adding $150 \mathrm{mg}$ of compound to $100 \mathrm{ml}$ of $0.9 \%$ normal saline and was infused slowly over 1 hour period. The woman was observed for: pulse rate, respiratory rate, blood pressure and fetal heart rate throughout and for another half an hour following the infusion for any untoward incident. Infusion was repeated every 3 days till total dose was completed.Test dose before the infusion was not done as it is not a reliable way to predict the patient response after full dose is given, still preparations were made to treat any untoward complications like anaphylactic shock.

Iron sorbitol citric acid complex was injected to group B women after sensitivity test as intramuscular injection of $1.5 \mathrm{ml}$ containing $75 \mathrm{mg}$ of elemental iron by using ' $\mathrm{Z}$ ' technique in upper and outer quadrant of buttock. Vitals were observed as for 30 minutes after the injection and the emergency kit was kept ready before each injection just in case of anaphylactic reaction took place. Daily injections were given till completion of the calculated dose. Patients in both the groups were followed on day 7, 21 and 30 after starting the iron therapy to check for hemoglobin level, hematocrit, MCV, MCH, MCHC and reticulocyte count. While on days 21 and 30 serum ferritin levels were also measured. The Z-track technique of intramuscular injection is used to prevent leakage of the medication into the subcutaneous tissue. During the procedure, skin in the upper and outer quadrant of buttock is pulled and held firmly while a long needle is inserted into the muscle. The medication is injected, slowly after it is confirmed that no blood vessel is entered, the skin is released. The needle track that forms during this procedure takes the shape of the letter "Z," which gives the procedure its name.

Z-track injection is usually less painful than a traditional IM injection. The Z-track method is particularly useful when a dark-colored drug that can cause staining of the skin is used. It also helps to prevent medication from seeping into the subcutaneous tissue and ensures a full dosage.

\section{Statistical analysis}

The data was analyzed by SPSS statistical software. chi square test was used to compare different parameters by converting them into categories. Student t-test was applied to compare mean levels of the parameters between the groups. To determine the statistical significance under each group of different parameters from baseline to different points of time $(7 / 21 / 30$ days), paired t test was used. 
Variations of $\mathrm{P}<0.05$ were considered to be statistically significant

\section{RESULTS}

Mean age of women in intravenous and intramuscular group was 24.8 and 25.6 years respectively. In-groupA, $47 \%$ cases were primigravida and the maximum parity 4 . In group $B$ there were $21 \%$ primigravida and the maximum parity was 5 , so significant difference.

Most of the patients $(60.5 \%)$ belonged to the lower middle class strata according to Indian socio-economical standards; only $12 \%$ were of upper middle and upper class strata. In group A, 9\% cases had hemoglobin between $5-6 \mathrm{gm} / \mathrm{dl}, 27 \%$ cases had between $6-7 \mathrm{gm} / \mathrm{dl}$ and $64 \% \mathrm{had}$ between 7-8 gm/ dl. In group B, $6 \%$ cases had hemoglobin levels between $5-6 \mathrm{gm} / \mathrm{dl}, 11 \%$ had between 6-7 gm/dl and $83 \%$ had between $7-8 \mathrm{gm} / \mathrm{dl}$ before the start of the therapy.

The mean hemoglobin level before the intravenous therapy was $7.24 \mathrm{gm} / \mathrm{dl}$ while the mean hemoglobin level before the start of the intramuscular therapy was $7.48 \mathrm{gm} / \mathrm{dl}$. (Figure 1).

Majority of the patients had requirement of iron between 1000-1400 mg in both the groups. The mean iron requirement was similar in both the groups (Figure 2).

The mean number of visits in-group $A$ was less as compared to group B (Figure 3).

The mean hemoglobin levels on $7^{\text {th }}$ day of starting the therapy in group A and group B were $8.154 \mathrm{gm} / \mathrm{dl}$ and $7.947 \mathrm{gm} / \mathrm{dl}$ respectively. On the $21^{\text {st }}$ day of therapy only $27 \%$ of cases in group B had attained haemoglobin level between $9-11 \mathrm{gm} / \mathrm{dl}$ while $78 \%$ cases in group A had attained haemoglobin levels of $9-11 \mathrm{gm} / \mathrm{dl}$.

Mean rise in haemoglobin level in-group B was less as compared to group A (Figure 4).

On day 30 of initiating the therapy $17 \%$ cases in intravenous group had attained the targeted haemoglobin level of $11 \mathrm{gm} / \mathrm{dl}$. However none of the patients in intramuscular group had attained the level of $11 \mathrm{gm} / \mathrm{dl}$.

Mean hemoglobin rise was 7.48, 7.947, 8.616 respectively after 7,21 and 30 days of inititing the therapy in the intramuscular group. Whereas in the intravenous group the rise in mean hemoglobin level was 8.154, 9.567 and 10.568 respectively after 7, 21 and 30 days. An earlier and faster

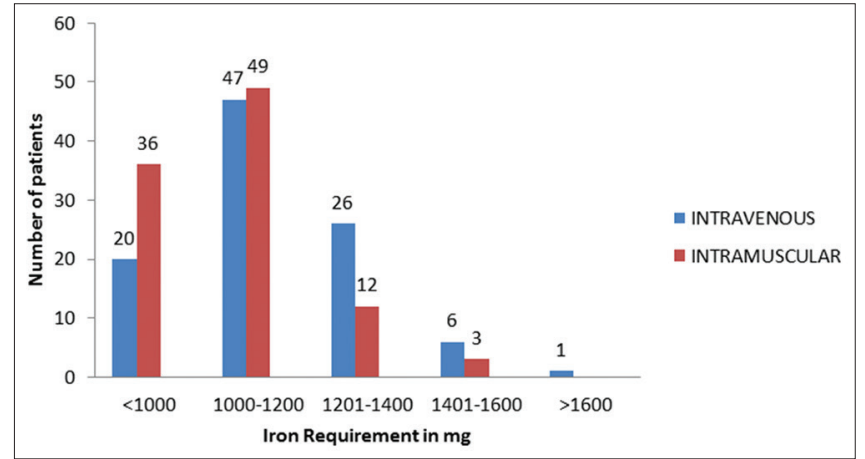

Figure 1: Iron requirements in patients

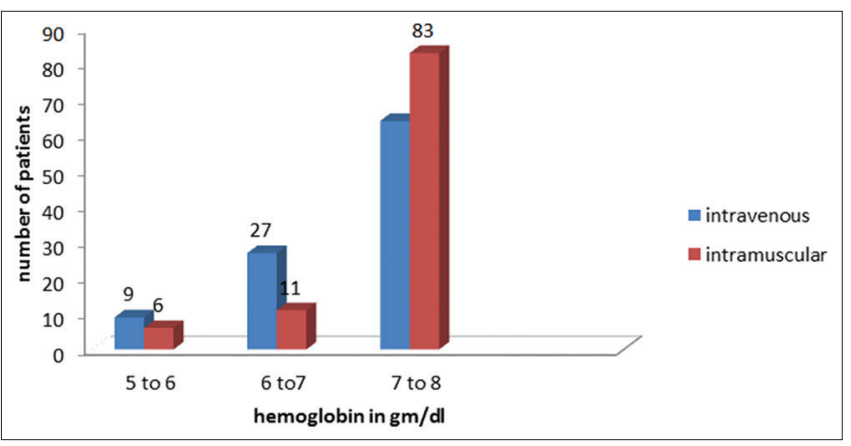

Figure 2: Hemoglobin levels before the start of therapy

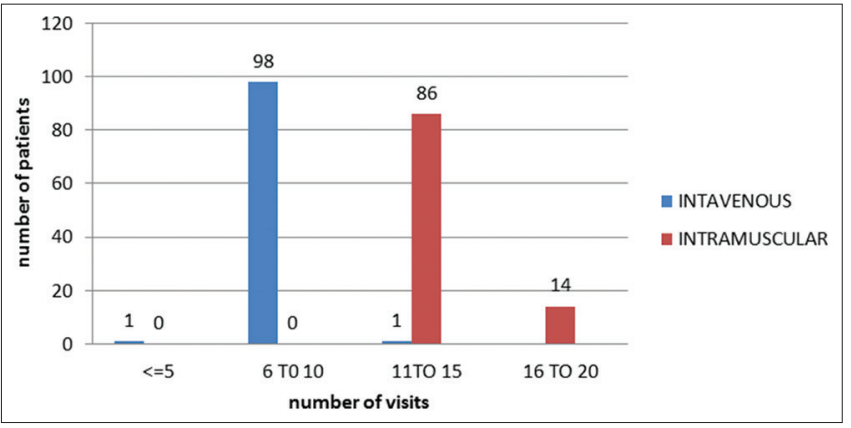

Figure 3: Number of hospital visits for iron therapy

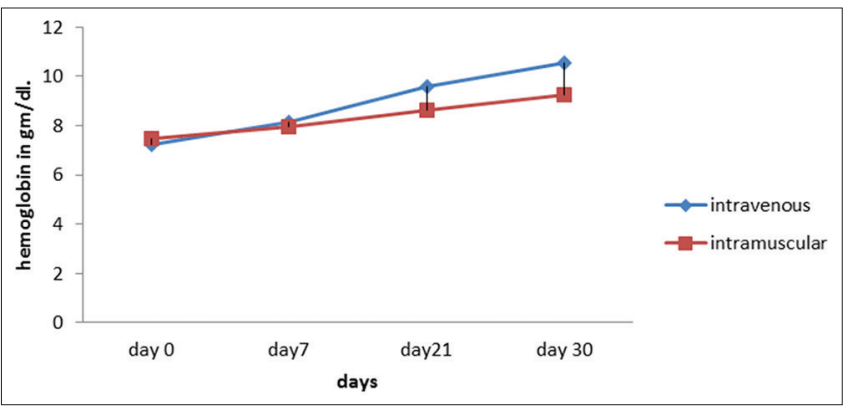

Figure 4: Comparison of hemoglobin levels after therapy

rise in hemoglobin was seen in the intravenous group in comparision to the intramuscular group. There was also a significant rise in PCV after therapy in the intravenous group as compared to the intramuscular group, especially on days 21 and 30 ( $p$ value $<0.05$ ). 
An increase in mean corpuscular volume of the red blood cells was seen in both the groups but was higher in the intravenous group on day 7 itself and a significantly higher mean corpuscular volume was achieved in the intravenous group on day 21 and 30 as compared to the intramuscular group. $(\mathrm{p}<0.005)$

There was significant rise in $\mathrm{MCH}$ and $\mathrm{MCHC}$ value in both the groups. However it was more in intravenous group. $(\mathrm{p}<0.05)$

An early response to therapy was evident by significant increase in the reticulocyte count in both the groups on day 7 itself, however the rise in reticulocyte count was significantly higher in the intravenous group as compared to the intramuscular group $(\mathrm{p}<0.005)$.

Serum ferritin levels in both the groups were comparable on day 0 , but the rising trend was more significant in the intravenous group as compared to other as shown by he following figures, rise in serum ferritin from mean of 10.526 on day 0 to 21.006 on day 21 and 33.758 on day 30 . In the intramuscular group rise from mean of 11.04 on day 0 to 14.633 on day 21 and 19.331 on day 30 was seen. $\mathrm{p}$ value was $<0.05$ on day 21 and 30 .

Pain at the local site and staining of skin were the most common side effects seen in the intramuscular therapy group (10-14\%). Fever, arthralgia, epigastric pain and headache were the other minor side effects noticed in 2-5\% of cases in intramuscular group. Shivering and phlebitis was noticed in 3-5\% of cases in intravenous group but did not warrant discontinuation of therapy. Other minor side effects like nausea and metallic taste was seen in only $1-2 \%$ of cases.

\section{DISCUSSION}

Iron dextran and iron gluconate as bolus injection, which was often prescribed earlier in our country limited the use of intravenous iron owing to the severe systemic adverse effects associated with these. Both iron dextran and iron gluconate cause unpredictable anaphylactic reactions and require a test dose before the first administration for treatment. Intravenous iron sucrose therapy is gaining popularity in recent years in our quest to treat moderate to severe iron deficiency anemia during pregnancy since it is claimed to be more effective and safe as compared to previously used parenteral iron preparations.

In our study most of the women were between the age group of 21-25 years, this being the reproductive age group in India and was comparable to the other studies. ${ }^{1,2,3}$ Sixty-six percentage of cases in our study were multigravida, which is comparable to other studies ${ }^{1,2,4}$ reason for this could be short interval between pregnancies even before iron deficit of previous pregnancy could be corrected resulting in iron deficiency anemia.

Women in the gestational age group 24-32 weeks were selected in this study as women with gestational age $<24$ weeks can be treated with oral iron therapy and those with gestational age $>32$ weeks do not have sufficient time to recover the haemoglobin deficit by means other than blood transfusion but transfusions have their own risks and costs so some intramuscular or intravenous iron can still be beneficial also because the iron can be used in the post partum period. However, Divakar $\mathrm{H}$ et $a l^{5}$ had recruited women within gestational age of 14-15 weeks because in this study a comparison was done between oral iron and intravenous iron sucrose and for oral therapy long follow up is required. Thirty-five point five percent of the women in our study had weight in the range of $46-55 \mathrm{~kg}$ which is comparable to the other studies done in this field. ${ }^{2,3,6}$ The rate of iron delivery to the marrow is a major factor in the regulation of marrow proliferation. Iron dextran and iron sucrose have different pharmacokinetic properties. Iron sucrose complex has an intermediate stability and strength. It is quickly cleared from serum with a half-life of approximately 5-6 hours compared with iron dextran, which has a serum half-life of 3-4 days. $3,7,8$

The iron sucrose consists of polynuclear iron complex analogous to ferritin with apoferritin component replaced by sucrose that is well tolerated and least antigenic and being a large molecule, less than $5 \%$ excreted by the kidneys. It is available for erythropoiesis within 5 minutes of infusion and has a $68-97 \%$ utilization rate after 2 - 4 weeks since it is stored in reticuloendothelial cells and not in the parenchymal cells of the body. The total iron required is calculated and administered, hence full dose administration is confirmed and is convenient to the patient because of limited number of visits. Besides, the goal of iron therapy i.e. to supply sufficient iron to correct hemoglobin deficit as well as to replenish stores is achieved without the need for further iron therapy throughout pregnancy and probably after. This compares favorably with iron sorbitol, which is painful and causes staining of the tissues, the absorption is unreliable and more than $30 \%$ is excreted via the kidneys in 4 hours. Within this time only a small amount is stored in liver and reticulo-endothelial cells while a relatively high fraction of iron complex deposits in parenchymal cells of liver, kidney and heart etc. Due to this reason a dose of $1.5 \mathrm{mg} / \mathrm{kg}$ cannot be exceeded which in a average $50 \mathrm{~kg}$ female accounts for $75 \mathrm{mg}$, compared to $7 \mathrm{mg} / \mathrm{kg}$ for iron sucrose which in a average $50 \mathrm{~kg}$ female accounts for $350 \mathrm{mg}$. In pregnancy the safe limit for iron sucrose is 
$200 \mathrm{mg}$. in a day. Earliest response to the therapy was seen by increase in the reticulocyte count in both the groups on day 7 of the therapy. None of the other studies have shown the response of reticulocyte count to intravenous and intramuscular therapy.

In intravenous group, 13\% women had grade I adverse effects and in intramuscular group, $40 \%$ cases had grade I adverse effects. These adverse effects were minimal and managed symptomatically. None of them required discontinuation of therapy in either group. None of the women had grade II adverse effects. While in a study by Wali ${ }^{6} 12 \%$ of IV group and $50 \%$ of IM group had grade 1 side effects.

Grade I side effects are mild to moderate reactions, not requiring discontinuation of therapy for e.g. local area pain, redness or swelling.

Grade II side effects are life threatening reactions, requiring discontinuation of therapy, e.g. severe anaphylactic reaction.

Limitations of intravenous iron could be in cases with acute blood loss as in these cases blood transfusion is preferred due to volume issue nevertheless the iron stores may be intact in such patients, but in patients with chronic anemia and with no contraindication to its use, intravenous iron therapy may be a better option in patients who have sufficient time left till delivery.

\section{CONCLUSIONS}

Intravenous iron sucrose therapy may prove a good alternative as an intermediate remedy when to choose between an ineffective oral iron therapy and the more aggressive and not risk free blood transfusion in patients who are not ideal candidates for either of the two therapeutic interventions in anemia during pregnancy. Efforts are going on in our country to explore this modality and to do extensive research on a large scale to achieve better results by introducing it in the national anemia prevention programme policy to replace oral iron during pregnancy which has being running for more than three decades with no respite seen in preventing development of anemia.

\section{REFERENCES}

1. Raja KS, Janjua NB and Khokhar N. Intravenous iron sucrose complex therapy in iron deficiency in the pregnant women. RMJ 2003; 28: 40-43.

2. Bayoumeu F, Subiran- Buisset C, Baka NE, Legagneur $H$, Monnier $\mathrm{P}$, Laxenaire $\mathrm{M}$, et al. Iron therapy in iron deficiency anaemia in pregnancy: intravenous route versus oral route. Am J Obstet Gynaecol 2002; 186: 518-522.

3. Hashmi Z, Ghazala B, Azeem P and Shah S. Effectiveness of intra-venous iron sucrose complex versus intra-muscular iron sorbitol in iron deficiency anemia. Ann Pak Inst Med Sci 2006; 2:188-189.

4. Agarwal $\mathrm{KN}$. Iron and brain: neurotransmitter receptors and magnetic resonance spectroscopy. Br J Nutr 2001; 85: S147-S150.

5. Divakar H, Nandakumar BS and Manyonda IT. Iron deficiency anemia in pregnancy: Is intravenous iron sucrose an alternative to the oral iron folate supplementation programme in India? 2009. Downloaded from http://www. abcofobg. com/IDA/docs.

6. Wali A, Mushtaq A and Nilofer. Comparative study-efficacy, safety and compliance of intravenous iron sucrose and intramuscular iron sorbitol in iron deficiency anemia of pregnancy. J Pak Med Assoc 2002; 52: 392-395.

7. Hunter J. Intramuscular injection techniques. Nursing Standard 2008: 22(24), 35-40.

8. Z-track injection (n.d). The Free Dictionary. Retrieved June 4, 2013 from http://medical-dictionary.thefreedictionary.com/Ztrack+injection.

\section{Author's contribution:}

HG-Concept and designed the study, scrutinized the overall project work from clinical and ethical point of view, prepared the first draft of manuscript

AS-Collected and compiled the data and carried out research project as part of dissertation; NS-Critically evaluated the contents, gave scientific inputs and

critically evaluated the manuscript.

Orcid ID:

Dr. Harsha Gaikwad:(1) http://orcid.org/0000-0003-0169-9346

Source of funding: Nil, Conflict of interest: None. 